PROCEEDINGS OF THE

AMERICAN MATHEMATICAL SOCIETY

Volume 128, Number 12, Pages 3675-3682

S 0002-9939(00)05471-X

Article electronically published on June 7, 2000

\title{
SOBOLEV TYPE INEQUALITIES FOR GENERAL SYMMETRIC FORMS
}

\author{
FENG-YU WANG
}

(Communicated by Stanley Sawyer)

\begin{abstract}
A general version of the Sobolev type inequality, including both the classical Sobolev inequality and the logarithmic Sobolev one, is studied for general symmetric forms by using isoperimetric constants. Some necessary and sufficient conditions are presented as results. The main results are illustrated by two examples of birth-death processes.
\end{abstract}

\section{INTRODUCTION}

The logarithmic Sobolev inequality has become a very active direction since initiated by Gross 7 in 1975. It has been well developed in the context of diffusions for both finite and infinite dimensional cases. There are also some results for finite Markov chains (see, e.g. 10] and references therein). In this paper, we study a general version of Sobolev inequalities for general symmetric forms by using isoperimetric constants. The main idea of the study goes back to Cheeger's inequality [3], which is well known and widely used in geometric analysis. This inequality is then established for bounded jump processes by Lawler and Sokal [8]. See also [6] for Cheeger's inequality for Markov chains on finite graphs. Recently, this inequality was also established by Chen and Wang [5] for general symmetric forms.

Let $(E, \mathcal{E})$ be a measurable space with a reference measure $\mu$ which is either a probability measure or is infinite. Next, let $J$ be a symmetric measure on $E \times E$. Define the symmetric form by

$$
\begin{aligned}
& D(f, g)=\frac{1}{2} \int_{E \times E} J(\mathrm{~d} x, \mathrm{~d} y)[f(y)-f(x)] \cdot[g(y)-g(x)], \\
& f, g \in \mathcal{D}:=\{h \in \mathcal{E}: D(h, h)<\infty\} .
\end{aligned}
$$

Let $F \in C(0, \infty)$ be such that $\inf r F(r)>-\infty$ and $F(r)>0$ for large $r$. We say that the $F$-Sobolev inequality with dimension $p \in(2, \infty]$ (denoted by $F S(p)$ ) holds for $(D, \mu)$ if there exist two constants $C_{1} \geq 0, C_{2}>0$ such that

$$
\mu\left(|f|^{2 p /(p-2)} F\left(f^{2}\right)\right)^{(p-2) / p} \leq C_{1}+C_{2} D(f, f)
$$

Received by the editors February 27, 1998 and, in revised form, September 21, 1998 and February 10, 1999.

1991 Mathematics Subject Classification. Primary 60J25, 47A75.

Key words and phrases. Sobolev inequality, isoperimetric constant, symmetric form.

The author's research was supported in part by the Alexander von Humboldt Foundation, NSFC(19631060), the Fok Ying-Tung Educational Foundation and the Research Foundation for Returned Overseas Chinese Scholars.

(C)2000 American Mathematical Society 
holds for all $f \in \mathcal{D}^{\prime}$ with $\mu\left(f^{2}\right)=1$, where $\mathcal{D}^{\prime}=\{f \in \mathcal{D}: \mu(f \neq 0)<\infty\}$. In particular, we call the inequality tight (denoted by $T F S(p)$ ) if (1.1) holds for $C_{1}=0$.

Obviously, when $F \equiv 1,(1.1)$ is just the classical Sobolev inequality with dimension $p$. When $\mu$ is a probability measure, $p=\infty$ and $F=\log$, (1.1) becomes the defective logarithmic Sobolev inequality, which is equivalent to the usual logarithmic Sobolev inequality

$$
\mu\left(f^{2} \log f^{2}\right) \leq C D(f, f), \quad \mu\left(f^{2}\right)=1, f \in \mathcal{D}^{\prime},
$$

whenever the spectral gap inequality holds; namely, there exists $\lambda_{1}>0$ such that

$$
\lambda_{1}\left[\mu\left(f^{2}\right)-\mu(f)^{2}\right] \leq D(f, f), \quad f \in \mathcal{D}^{\prime} .
$$

We now look for a proper isoperimetric constant which is hoped to best describe $F S(p)$. We shall need the following condition of $F$ :

$$
F \in C^{1}[0, \infty), F(0)=1, F^{\prime} \geq 0 \text { and } \lim _{r \rightarrow \infty} \sup \frac{(p-2) r F^{\prime}(r)}{2 p F(r)}<\frac{p-1}{p} .
$$

Moreover, it is natural to assume the following as soon as $F S(p)$ is considered:

$$
\inf \{\mu(A): \mu(A)>0\}=0 .
$$

Otherwise, $\mu\left(f^{2}\right)=1$ implies that $f^{2} \leq C$ a.e.- $\mu$ for some constant $C$; then one has $F S(p)$ for any $p$ and $F$. Under (1.5), and assuming that $\lim _{r \rightarrow \infty} F(r)=\infty$ whenever $F S(\infty)$ is considered, by taking the test function $f=1_{A} / \sqrt{\mu(A)}$ in (1.1) and then letting $\mu(A) \rightarrow 0$, one immediately sees that $F S(p)$ implies $\kappa_{p}(0)>0$. Similarly, $T F S(p)$ implies $\kappa_{p}(\infty)>0$, where

$$
\begin{aligned}
& \kappa_{p}(r)=\inf _{\mu(A) \in(0, r]} \frac{J\left(A \times A^{c}\right)}{\mu(A)^{(p-2) / p} F\left(\mu(A)^{-1}\right)^{(p-2) / p}}, \quad r>0, \\
& \kappa_{p}(0)=\lim _{r \rightarrow 0+} \kappa_{p}(r), \quad \kappa_{p}(\infty)=\lim _{r \rightarrow \infty} \kappa_{p}(r) .
\end{aligned}
$$

Consequently, if (1.5) holds and $J(\mathrm{~d} x, E) \leq M \mu(\mathrm{d} x)$ for some constant $M$, then $F S(p)$ does not hold (we assume in addition $\lim _{r \rightarrow \infty} F(r)=\infty$ when $p=\infty$ ). Actually, in this case, by letting $\mu(A) \rightarrow 0$, one has $\kappa_{p}(0)=0$. The problem then becomes whether " $\kappa_{p}(0)>0$ " is also sufficient for $F S(p)$. Unfortunately, the answer is negative. Actually, it is not difficult to give some counterexamples. See e.g. Example 3.1 in section 3 .

But what is the correct constant to ensure $F S(p)$ ? It should be in some sense smaller than the one defined above. So, it is natural to define the constant by using a "smaller" symmetric measure instead of $J$. To do this, we follow the line of [5]. Let $\gamma$ be a measurable nonnegative symmetric function such that

$$
\int_{A \times E} \frac{1_{\{\gamma(x, y)>0\}} J(\mathrm{~d} x, \mathrm{~d} y)}{\gamma(x, y)} \leq \mu(A), \quad A \in \mathcal{E} .
$$

Define $J^{\prime}(\mathrm{d} x, \mathrm{~d} y)=1_{\{\gamma(x, y)>0\}} J(\mathrm{~d} x, \mathrm{~d} y) / \sqrt{\gamma(x, y)}$ and

$$
\begin{aligned}
& k_{p}(r)=\inf _{\mu(A) \in(0, r]} \frac{J^{\prime}\left(A \times A^{c}\right)}{\mu(A)^{(p-1) / p} F\left(\mu(A)^{-1}\right)^{(p-2) / 2 p}}, \quad r>0, \\
& k_{p}(0)=\lim _{r \rightarrow 0+} k_{p}(r), \quad k_{p}(\infty)=\lim _{r \rightarrow \infty} k_{p}(r) .
\end{aligned}
$$

Note that the parameter $p$ takes different roles in the definitions of $\kappa_{p}$ and $k_{p}$, where the former comes from our choice of test functions and the latter is due to 
the proof of Theorem 1.1. According to the isoperimetric constants for Sobolev inequalities on manifolds (see e.g. [3]), one may feel that the latter is more natural. Actually, as will be shown in Example $3.1, k_{p}$ can describe $F S(p)$ precisely.

It is now time to state our main result.

Theorem 1.1 (Under (1.4)). If $k_{p}(0)>0$ (resp. $\left.k_{p}(\infty)>0\right)$, then $F S(p)$ (resp. TFS $(p))$ holds. In particular, if $\mu$ is a probability measure, then the logarithmic Sobolev inequality (1.2) holds provided $k_{\infty}(1 / 2)>0$ for $F(r)=\log (r+\mathrm{e})$.

Before moving further, let us recall some concrete cases of the form $D$, or equivalently, the measure $J$. For a symmetric jump process (see e.g. 4]), we have a $q$-pair $(q(x), q(x, \mathrm{~d} y))$. We assume in this paper that $q(x)=q(x, E \backslash\{x\})<\infty$. Let $\mu$ be the symmetric measure (not necessarily finite); we have $J(\mathrm{~d} x, \mathrm{~d} y)=q(x, \mathrm{~d} y) \mu(\mathrm{d} x)$. In this case, a natural choice of $\gamma$ is $\gamma(x, y)=q(x) \vee q(y)$. When $E$ is countable, we simply denote $q_{i j}=q(i,\{j\}), q_{i}=q(i)$. Especially, we shall often consider the most simple but very interesting case; namely, a birth-death process: $E=\mathbb{Z}_{+}, q_{i, i-1}=a_{i}$ (the death rate), $q_{i, i+1}=b_{i}$ (the birth rate) and $q_{i j}=0$ for $|i-j| \neq 1$. Note that in the final case we always assume $a_{0}=0$.

We present below a criterion of $k_{p}$ for countable Markov chains.

Theorem 1.2 (Under (1.4)). Consider the countable Markov chain: $E=\mathbb{Z}_{+}$and $J(i, j)=q_{i j} \mu_{i}$ with $\mu_{i}>0(i \geq 0)$ and $\sum_{i} \mu_{i}=1$. Let $\gamma(i, j)=q_{i} \vee q_{j}, q_{i j}^{\prime}=$ $q_{i j} / \sqrt{\gamma(i, j)}$ and $I_{i}=[i, \infty) \cap E$. We have $k_{p}(0)>0$ provided

$$
\inf _{i \geq 1} \frac{\mu_{i} \sum_{j=0}^{i-1} q_{i j}^{\prime}}{\mu\left(I_{i}\right)^{(p-1) / p} F\left(\mu\left(I_{i}\right)^{-1}\right)^{(p-2) / 2 p}}>0 .
$$

Moreover, (1.7) is also necessary for irreducible birth-death processes (i.e. $a_{i}, b_{i-1}>$ 0 for all $i \geq 1)$. Similarly, the result also holds for $\kappa_{p}$ with $q_{i j}^{\prime}$ replaced by $q_{i j}$.

We have pointed out that $\kappa_{p}(0)>0$ is usually weaker than $F S(p)$. A natural question is: can we prove a weaker inequality from $\kappa_{p}(0)>0$ ? The answer is positive and the inequality is the following:

$$
\mu\left(|f|^{2 p /(p-2)} F\left(f^{2}\right)\right)^{(p-2) / p} \leq C_{1}+C_{2} \int J(\mathrm{~d} x, \mathrm{~d} y)\left|f(x)^{2}-f(y)^{2}\right|,
$$

where $f \in \mathcal{D}^{\prime}$ with $\mu\left(f^{2}\right)=1$. We simply denote it by $W F S(p)$ or $W T F S(p)$ in terms of $C_{1}>0$ or $C_{1}=0$. Obviously, (1.1) implies (1.1') and they are equivalent when $J(E, \mathrm{~d} x) \leq M \mu(\mathrm{d} x)$ for some constant $M$.

Theorem 1.3. Assume that $F \geq 0, F^{\prime} \geq 0$ and $\sup \frac{r F^{\prime}(r)}{F(r)}<\infty$. Moreover, assume that $F(\infty)=\infty$ whenever the case " $p=\infty$ and $C_{1}>0$ " is considered. We have $W F S(p)($ resp. WTFS $(p))$ if and only if $\kappa_{p}(0)>0\left(\right.$ resp. $\left.\kappa_{p}(\infty)>0\right)$.

Finally, we modify Herbst's argument to study the exponential integrability of "Lipschitz functions" under the logarithmic Sobolev inequality. See 1] (and also 9]) for detailed discussions in the context of diffusions.

Let $\rho \geq 0$ be a measurable function on $E$. Assume that

$$
|\rho(x)-\rho(y)|^{2} \gamma(x, y) \leq 1 \quad \text { a.e.- } J,
$$

where $\gamma$ is as in (1.6). In particular, when $J(\mathrm{~d} x, \mathrm{~d} y)=q(x, \mathrm{~d} y) \mu(\mathrm{d} x),(1.8)$ implies

$$
\int|\rho(x)-\rho(y)|^{2} q(x, \mathrm{~d} y) \leq 1 \quad \text { a.e. }-\mu \text {. }
$$


Theorem 1.4. Assume that $\rho(\geq 0)$ satisfies (1.8) (more weakly, (1.9) for the case $J(\mathrm{~d} x, \mathrm{~d} y)=q(x, \mathrm{~d} y) \mu(\mathrm{d} x))$. If (1.2) holds, then

$$
\mu\left(\mathrm{e}^{\varepsilon \rho^{2}}\right) \leq \exp \left[\frac{\varepsilon \mu\left(\rho^{2}\right)}{1-\varepsilon C}\right]<\infty, \quad \varepsilon \in[0,1 / C) .
$$

We remark that according to [5], (1.3) implies that $\mu\left(\mathrm{e}^{\alpha \rho}\right)<\infty$ for some $\alpha>0$. Hence $\mu\left(\rho^{2}\right)<\infty$ under $(1.2)$.

The above results are proved in the next section and are illustrated in section 3 by two examples of birth-death processes.

\section{Proofs}

Proof of Theorem 1.1. Since $D(|f|,|f|) \leq D(f, f)$, it suffices to prove for the case $f \geq 0$. Moreover, we assume that $f \in \mathcal{D}^{\prime}$ is bounded so that each term in (1.1) is finite. If $f \geq 0$ is unbounded, we need only to replace $f$ by $f \wedge n$ and then let $n \rightarrow \infty$.

For a bounded nonnegative $f \in \mathcal{D}^{\prime}$ with $\mu\left(f^{2}\right)=1$, let $h(t)=\mu\left(f^{2}>t\right)$. Set $\phi(t)=t^{(p-1) /(p-2)} \sqrt{F(t)}$ which is strictly increasing under (1.4). Take $g=$ $\phi\left(f^{2}\right), A_{t}=\{g>t\}$ and $\bar{h}(t)=\mu\left(A_{t}\right)$. If $k_{p}(r)>0$ for some $r>0$, we have $\bar{h}(t)=h\left(\phi^{-1}(t)\right) \leq 1 / \phi^{-1}(t) \leq r$ for $t \geq \phi\left(r^{-1}\right)$. Then, by the isoperimetric inequality and Fubini's theorem, we have

$$
\begin{aligned}
I & :=\left\{\frac{1}{2} \int J^{\prime}(\mathrm{d} x, \mathrm{~d} y)|g(x)-g(y)|\right\}^{p /(p-1)} \\
& =\left\{\int_{\{g(x)>g(y)\}} J^{\prime}(\mathrm{d} x, \mathrm{~d} y)(g(x)-g(y))\right\}^{p /(p-1)} \\
& =\left\{\int_{0}^{\infty} J^{\prime}\left(A_{t} \times A_{t}^{c}\right) \mathrm{d} t\right\}^{p /(p-1)} \\
& \geq\left\{k_{p}(r) \int_{\phi\left(r^{-1}\right)}^{\infty} \bar{h}(t)^{(p-1) / p} F\left(\bar{h}(t)^{-1}\right)^{(p-2) / 2 p} \mathrm{~d} t\right\}^{p /(p-1)} \\
& =c_{1} \int_{\phi\left(r^{-1}\right)}^{\infty}\left\{\int_{\phi\left(r^{-1}\right)}^{t} \bar{h}(s)^{(p-1) / p} F\left(\bar{h}(s)^{-1}\right)^{(p-2) / 2 p} \mathrm{~d} s\right\}^{1 /(p-1)} \\
& \bar{h}(t)^{(p-1) / p} F\left(\bar{h}(t)^{-1}\right)^{(p-2) / 2 p} \mathrm{~d} t,
\end{aligned}
$$

where $c_{1}=\left[k_{p}(r)\right]^{p /(p-1)} p /(p-1)$.

Let $\xi(t)=t^{(p-1) / p} F\left(t^{-1}\right)^{(p-2) / 2 p}$; we have

$$
\xi^{\prime}(t)=t^{-1 / p} F\left(t^{-1}\right)^{(p-2) / 2 p}\left(\frac{p-1}{p}-\frac{(p-2) F^{\prime}\left(t^{-1}\right)}{2 p t F\left(t^{-1}\right)}\right) .
$$

Then, by (1.4), $\xi^{\prime}(t)>0$ for both small $t$ and big $t$. Therefore, there exists $c_{2}>0$ such that $\xi(t) \geq c_{2} \xi(s)$ for all $t \geq s \geq 0$. Taking this into account and noting that 
$h$ is decreasing with $h(t) \leq 1 / t$, by (2.1) we obtain

$$
\begin{aligned}
I & \geq c_{3} \int_{\phi\left(r^{-1}\right)}^{\infty}\left[t-\phi\left(r^{-1}\right)\right]^{1 /(p-1)} \bar{h}(t) F\left(\bar{h}(t)^{-1}\right)^{(p-2) / 2(p-1)} \mathrm{d} t \\
& =c_{3} \int_{r^{-1}}^{\infty}\left[\phi(t)-\phi\left(r^{-1}\right)\right]^{1 /(p-1)} h(t) F\left(h(t)^{-1}\right)^{(p-2) / 2(p-1)} \phi^{\prime}(t) \mathrm{d} t \\
& \geq c_{3} \int_{r^{-1}}^{\infty}\left[\phi(t)-\phi\left(r^{-1}\right)\right]^{1 /(p-1)} F(t)^{(p-2) / 2(p-1)} \phi^{\prime}(t) h(t) \mathrm{d} t
\end{aligned}
$$

for some $c_{3}>0$, where the second step is due to the integral transformation $t \rightarrow$ $\phi(t)$.

Now, for each $r \in(0, \infty)$, take $\beta(r) \geq 0$ such that for all $t \geq r^{-1}$,

$$
\begin{aligned}
{[\phi(t)} & \left.-\phi\left(r^{-1}\right)\right]^{1 /(p-1)} F(t)^{(p-2) / 2(p-1)} \phi^{\prime}(t) \\
& \geq \frac{1}{2} \phi(t)^{1 /(p-1)} F(t)^{(p-2) / 2(p-1)} \phi^{\prime}(t)-\beta(r) .
\end{aligned}
$$

Moreover, we may take $\beta(\infty)=0$ for $r=\infty$. We then obtain

$$
\begin{aligned}
I & \geq \frac{c_{3}}{2} \int_{1 / r}^{\infty} \phi(t)^{1 /(p-1)} F(t)^{(p-2) / 2(p-1)} \phi^{\prime}(t) h(t) \mathrm{d} t-c_{3} \beta(r) \\
& \geq \frac{c_{3}}{2} \int_{0}^{\infty} \phi(t)^{1 /(p-1)} F(t)^{(p-2) / 2(p-1)} \phi^{\prime}(t) h(t) \mathrm{d} t-c(r),
\end{aligned}
$$

where $c(r)=c_{3} \beta(r)+\frac{c_{3}}{2} \sup _{\left(0, r^{-1}\right)} \phi(t)^{1 /(p-1)} F(t)^{(p-2) / 2(p-1)} \phi^{\prime}(t)$ which is zero when $r=\infty$. Let

$$
\psi(t)=\frac{\mathrm{d}}{\mathrm{d} t}\left\{t^{p /(p-2)} F(t)\right\} .
$$

By (1.4), there exists $c^{\prime}>0$ such that

$$
\phi(t)^{1 /(p-1)} F(t)^{(p-2) / 2(p-1)} \phi^{\prime}(t) \geq \frac{p-1}{p-2} t^{2 /(p-2)} F(t) \geq c^{\prime} \psi(t) .
$$

Therefore, there exists $c_{4}>0$ such that

$$
\begin{aligned}
I+c(r) & \geq c_{4} \int_{0}^{\infty} \psi(t) h(t) \mathrm{d} t=c_{4} \int_{E} \mathrm{~d} \mu \int_{0}^{f^{2}} \psi(t) \mathrm{d} t \\
& =c_{4} \mu\left(f^{2 p /(p-2)} F\left(f^{2}\right)\right) .
\end{aligned}
$$

On the other hand, let $\eta(t)=\phi\left(t^{2}\right)$; we have

$$
|g(x)-g(y)|=|f(x)-f(y)| \cdot\left|\eta^{\prime}(\theta)\right|
$$

for some $\theta \in[f(x) \wedge f(y), f(x) \vee f(y)]$. It is easy to see that $\left|\eta^{\prime}(t)\right| \leq c_{5} \eta(t) / t$ for some $c_{5}>0$. Since $\eta(t) / t$ is increasing in $t$,

$\left|\eta^{\prime}(\theta)\right| \leq \frac{c_{5} \eta(f(x) \vee f(y))}{f(x) \vee f(y)} \leq c_{5}\left\{f(x)^{p /(p-2)} \sqrt{F\left(f(x)^{2}\right)}+f(y)^{p /(p-2)} \sqrt{F\left(f(y)^{2}\right)}\right\}$.

Therefore, by the Schwartz inequality and using (1.6), we obtain

$$
I \leq c_{6} \sqrt{D(f, f) \mu\left(f^{2 p /(p-2)} F\left(f^{2}\right)\right)} .
$$

The proof of the first assertion is now completed by combining this with (2.2). Just note that when $k_{p}(\infty)>0$, we may take $r=\infty$ and hence $c(r)=0$. 
Finally, if $\mu$ is a probability measure and $k_{\infty}(1 / 2)>0$ for $F(r)=\log (r+\mathrm{e})$, then the defective logarithmic Sobolev inequality holds. On the other hand, by [5, Theorem 1.2], $k_{\infty}(1 / 2)>0$ implies (1.3) for some $\lambda_{1}>0$. Therefore (1.2) holds.

Proof of Theorem 1.2. For any $A$ with $\mu(A)<\mu_{0}$, we have $0 \notin A$. Let $i=\inf A$, then $i \geq 1$. Noting that $t^{(p-1) / p} F\left(t^{-1}\right)^{(p-2) / 2 p}$ is increasing for small and big $t$, we have

$$
\frac{J^{\prime}\left(A \times A^{c}\right)}{\mu(A)^{(p-1) / p} F\left(\mu(A)^{-1}\right)^{(p-2) / 2 p}} \geq \frac{c \mu_{i} \sum_{j<i} q_{i j}^{\prime}}{\mu\left(I_{i}\right)^{(p-1) / p} F\left(\mu\left(I_{i}\right)^{-1}\right)^{(p-2) / 2 p}}
$$

for some constant $c>0$. This implies $k_{p}(0)>0$ by (1.7).

On the other hand, we consider irreducible birth-death processes. If $k_{p}(0)>0$, then there exists $r \in(0,1 / 2)$ such that $k_{p}(r)>0$. For any $i$ with $\mu\left(I_{i}\right) \leq r$, we have

$$
\frac{\mu_{i} q_{i, i-1}^{\prime}}{\mu\left(I_{i}\right)^{(p-1) / p} F\left(\mu\left(I_{i}\right)^{-1}\right)^{(p-2) / 2 p}}=\frac{J^{\prime}\left(I_{i} \times I_{i}^{c}\right)}{\mu\left(I_{i}\right)^{(p-1) / p} F\left(\mu\left(I_{i}\right)^{-1}\right)^{(p-2) / 2 p}} \geq k_{p}(r)>0 .
$$

Therefore (1.7) holds.

Proof of Theorem 1.3. It suffices to prove the sufficiency. Assume that $\kappa_{p}(r)>0$ for some $r>0$ and let $f$ and $h$ be as in the proof of Theorem 1.1. We then have, as was shown in the proof of Theorem 1.1,

$$
\begin{aligned}
& \left\{\frac{1}{2} \int J(\mathrm{~d} x, \mathrm{~d} y)\left|f(x)^{2}-f(y)^{2}\right|\right\}^{p /(p-2)} \\
& \geq\left\{\kappa_{p}(r) \int_{r^{-1}}^{\infty} h(t)^{(p-2) / p} F\left(h(t)^{-1}\right)^{(p-2) / p} \mathrm{~d} t\right\}^{p /(p-2)} \\
& \geq c \int_{0}^{\infty} t^{2 /(p-2)} F(t) h(t) \mathrm{d} t-c(r) \\
& \geq c^{\prime} \int_{0}^{\infty} \frac{\mathrm{d}}{\mathrm{d} t}\left\{t^{p /(p-2)} F(t)\right\} h(t) \mathrm{d} t-c(r) \\
& =\mu\left(f^{2 p /(p-2)} F\left(f^{2}\right)\right)-c(r)
\end{aligned}
$$

for some $c, c^{\prime}>0$ and $c(r) \geq 0$ with $c(\infty)=0$. The proof is then finished.

Proof of Theorem 1.4. The proof is essentially modified from 11. For any $n \geq 1$, let $\rho_{n}=\rho \wedge n, f_{n}=\exp \left[r \rho_{n}^{2} / 2\right]$ and $h_{n}(r)=\mu\left(\mathrm{e}^{r \rho_{n}^{2}}\right)$. By (1.8) we have

$$
\begin{aligned}
D\left(f_{n}, f_{n}\right) & \leq \frac{r^{2}}{2} \int\left|\rho_{n}(x)-\rho_{n}(y)\right|^{2} \max \left\{\rho_{n}(x)^{2} f_{n}(x)^{2}, \rho_{n}(y)^{2} f_{n}(y)^{2}\right\} J(\mathrm{~d} x, \mathrm{~d} y) \\
& \leq r^{2} \mu\left(\rho_{n}^{2} \mathrm{e}^{r \rho_{n}^{2}}\right)=r^{2} h_{n}^{\prime} .
\end{aligned}
$$

Similarly, since $J$ is symmetric, if $J(\mathrm{~d} x, \mathrm{~d} y)=q(x, \mathrm{~d} y) \mu(\mathrm{d} x)$ and (1.9) holds, then the above estimate remains true. By this and (1.2) we obtain

$$
r h_{n}^{\prime}(r) \leq h_{n}(r) \log h_{n}(r)+r^{2} C h_{n}^{\prime}(r), \quad r \geq 0 .
$$

The remainder of the proof is similar to that of [1, Theorem 3.3]. 


\section{EXAMPLES}

In the following two examples, we take $F(r)=[\log (r+\mathrm{e})]^{\delta}, \delta \geq 0$.

Example 3.1. Consider the birth-death process with $a_{i}=b_{i}=i^{s}[\log (i+1)]^{t}(i \geq$ $1), s>1, t \in \mathbb{R}$ and $a_{0}=0, b_{0}=1$. We have $\mu_{i}=m_{i} / c, m_{0}=1, m_{i}=$ $i^{-s}[\log (i+1)]^{-t}, c=\sum_{i} m_{i}$. Then $\kappa_{p}(0)>0$ for all $p$ and $\delta$. Assume that $\delta>0$ whenever $p=\infty$ is considered; then $F S(p)$ holds if and only if either $s>2(p-1) /(p-2)$, or $s=2(p-1) /(p-2)$ and $t \geq \delta$.

Especially, the logarithmic Sobolev inequality holds if and only if either $s>2$, or $s=2$ and $t \geq 1$.

Proof. $\kappa_{p}(0)>0$ is obvious. Actually, by the same spirit of Theorem 1.2, it follows from the fact that (noting that $\mu_{i} a_{i}=1 / c$ )

$$
\inf _{i \geq 1} \frac{\mu_{i} a_{i}}{\mu\left(I_{i}\right)^{(p-2) / p}\left[\log \left(\mu\left(I_{i}\right)^{-1}+\mathrm{e}\right)\right]^{\delta(p-2) / p}}>0 .
$$

Next, for $i>>1$, we have

$$
\begin{aligned}
& \mu\left(I_{i}\right)^{(p-1) / p} F\left(\mu\left(I_{i}\right)^{-1}\right)^{(p-2) / 2 p}=O\left(i^{(1-s)(p-1) / p}[\log (i+1)]^{\delta(p-2) / 2 p-t(p-1) / p}\right), \\
& \frac{a_{i} \mu_{i}}{\sqrt{q_{i} \vee q_{i-1}}}=O\left(i^{-s / 2}[\log (i+1)]^{-t / 2}\right) .
\end{aligned}
$$

Therefore, (1.7) holds if either $s>2(p-1) /(p-2)$, or $s=2(p-1) /(p-2)$ but $t \geq \delta$.

Now, we go to prove the necessity case by case.

1 ) Assume that $s=2(p-1) /(p-2)$ and $t<\delta$. For any $n \geq 1$, take $f_{n}(i)=$ $i 1_{\{i \leq n\}}$. Then, for big $n$,

$$
\frac{D\left(f_{n}, f_{n}\right)+\mu\left(f_{n}^{2}\right)}{\mu\left(f_{n}^{2 p /(p-2)}\left[\log \left(f^{2}+\mathrm{e}\right)\right]^{\delta}\right)}=O\left(\frac{n+\sum_{i=1}^{n} i^{2-2(p-1) /(p-2)}[\log (i+1)]^{-t}}{\left(\sum_{i=1}^{n} i^{2 /(p-2)}[\log (i+1)]^{\delta-t}\right)^{(p-2) / p}}\right)
$$

which goes to zero as $n \rightarrow \infty$ (noting that $\delta>0$ for $p=\infty$ ). Hence $F S(p)$ does not hold.

2) Assume that $p<\infty$ and $s<2(p-1) /(p-2)$. Then $(s-1)(p-2) / 2 p<$ $\min \{1 / 2,(s-1) / 2\}$. Choose $\delta^{\prime} \in((s-1)(p-2) / 2 p, \min \{1 / 2,(s-1) / 2\})$ and take $f(i)=i^{\delta^{\prime}}$. We have, for some $c_{1}, c_{2}>0$,

$$
\mu\left(f^{2}\right) \leq c_{1} \sum_{i} i^{-s+2 \delta^{\prime}}\left[\log [(i+1)]^{-t}<\infty, \quad D(f, f) \leq c_{1} \sum_{i} i^{2\left(\delta^{\prime}-1\right)}<\infty\right.
$$

but (noting that $2 p \delta^{\prime} /(p-2)-s>-1$ )

$$
\mu\left(f^{2 p /(p-2)}\left[\log \left(f^{2}+\mathrm{e}\right)\right]^{\delta}\right) \geq c_{2} \sum_{i} i^{2 p \delta^{\prime} /(p-2)-s}[\log (i+1)]^{-t+\delta}=\infty .
$$

Hence $F S(p)$ does not hold.

3) Assume that $p=\infty, \delta>0$ and $s<2$. Let $f_{n}=\sqrt{i} 1_{\{i \leq n\}}$. We find that

$$
\frac{D\left(f_{n}, f_{n}\right)+\mu\left(f_{n}^{2}\right)}{\mu\left(f_{n}^{2}\left[\log \left(f_{n}^{2}+\mathrm{e}\right)\right]^{\delta}\right)}=O\left(\frac{\sum_{i=1}^{n} i^{1-s}[\log (i+1)]^{-t}}{\sum_{i=1}^{n} i^{1-s}[\log (i+1)]^{\delta-t}}\right),
$$

which goes to zero as $n \rightarrow \infty$. Therefore, $F S(\infty)$ does not hold.

Finally, for the assertion on the logarithmic Sobolev inequality (1.2), it suffices to prove that (1.7) implies (1.3). This is true for irreducible birth-death processes as claimed by [5, Theorem 4.1]. 
Example 3.2. Consider the birth-death process with $a_{0}=0, a_{i}=1(i \geq 1), b_{i}=$ $\mu_{i+1} / \mu_{i}(i \geq 0)$ and $\mu_{0}=1, \mu_{i}=i^{s}[\log (i+1)]^{t}$. Then TFS $(p)$ holds provided either $s \geq p-1$, or $s=p-1$ and $t \geq 0$. It is not strange that $\delta$ does not appear in the condition, because here $\mu_{i}$ has positive lower bound and hence the positivity of the constant $k_{p}(\infty)$ is independent of $\delta$.

Proof. Let $A \in \mathcal{E}$ with $\mu(A)<\infty$. We have $i:=\sup A<\infty$. Noting that $\gamma(i, j)=$ $q_{i} \vee q_{j}$ is bounded, we have

$$
J^{\prime}\left(A \times A^{c}\right) \geq c_{1} i^{s}[\log (i+1)]^{t}, \quad \mu(A) \leq \sum_{j \leq i} \mu_{j} \leq c_{2} i^{s+1}[\log (i+1)]^{t} .
$$

Therefore,

$$
\frac{J^{\prime}\left(A \times A^{c}\right)}{\mu(A)^{(p-1) / p}\left[\log \left(\mu(A)^{-1}+\mathrm{e}\right)\right]^{\delta}} \geq c_{3} i^{s-(s+1)(p-1) / p}[\log (i+\mathrm{e})]^{t / \delta}
$$

for some $c_{3}>0$. By Theorem 1.2, under our conditions we have $k_{p}(\infty)>0$ which implies $T F S(p)$ according to Theorem 1.1.

\section{ACKNOWLEDGEMENTS}

The author would like to thank Professors M. F. Chen, S. A. Sawyer and the referee for their careful comments on the first version of the paper. The revised version was also stimulated by conversations with Professor D. Bakry when the author visited him in Toulouse from April to June 1998.

\section{REFERENCES}

1. Aida, S., Musuda, T. and Shigekawa, I., Logarithmic Sobolev inequalities and exponential integrability, J. Funct. Anal. 126(1994), 83-101. MR 95m:60111

2. Chavel, I., Eigenvalues in Riemannian Geometry, New York: Academic Press, 1984. MR 86g:58140

3. Cheeger, J., A lower bound for the smallest eigenvalue of the Laplacian, Problems in Analysis, a symposium in honor of S. Bochner 195-199, Princeton U. Press, Princeton. MR 53:6645

4. Chen, M. F., From Markov Chains to Non-Equilibrium Particle Systems, Singapore: World Scientific, 1992. MR 94a:60135

5. Chen, M. F. and Wang, F. Y., Cheeger's inequalities for general symmetric forms and existence criteria for spectral gap, MSRI Preprint, 1998-024, to appear in Ann. Probab. CMP 99:07

6. Chung, F. R. F., Spectral Graph Theory, GBMS, 92, AMS, Providence, Rhode Island, 1997. MR 97k:58183

7. Gross, L., Logarithmic Sobolev inequalities, Amer. J. Math. 97(1975), 1061-1083. |MR 54:8263

8. Lawler, G. F. and Sokal, A. D., Bounds on the $L^{2}$ spectrum for Markov chains and Markov processes: generalization of Cheeger's inequality, Trans. Amer. Math. Soc. 309(1998), 557580. MR 89h:60105

9. Ledoux, M., Concentration of measure and logarithmic Sobolev inequalities, 1997 preprint.

10. Saloff-Coste, L., Lectures on finite Markov Chains, Lecture Notes in Math. 1665(1997), 301413. MR 99b:60119

Department of Mathematics, Beijing Normal University, Beijing 100875, People's Republic of China

E-mail address: wangfy@bnu.edu.cn

Current address: Universität Bielefeld, Fakultät für Mathematik, Postfach 100131, D-33501

Bielefeld, Germany

E-mail address: fwang@mathematik.uni-bielefeld.de 